\title{
EXPERIENCIAS
}

\section{COLABORACION ESCUELA-FAMILIAS: ANÁLISIS DE UNA EXPERIENCIA DE ESCUELA DE PADRES EN EL I.E.S. TORREBLANCA DE SEVILLA DURANTE EL CURSO 1998/99}

\author{
SCHOOL-FAMILIES COOPERATION: ANALYSIS OF A PRACTICAL \\ EXPERIENCE AT THE «TORREBLANCA» HIGH SCHOOL IN SEVILLE \\ DURING THE ACADEMIC YEAR 1989/99
}

\author{
Manuel Moreno* \\ Orientador escolar
}

\begin{abstract}
RESUMEN
Con la presente experiencia, pretendemos potenciar la comunicación y el diálogo, de modo permanente y comprensivo entre profesorado y familias en nuestro contexto educativo. El planteamiento fue coordinado por el orientador del centro, contando con el apoyo y respaldo de la Dirección del mismo, la colaboración y participación de un grupo de profesores y sobre todo de las familias que se implicaron en ella.

Se intentó crear un espacio de reflexión sobre los conflictos cotidianos, ofertando soluciones, alternativas, criterios de actuación y consiguiendo un mejor entendimiento entre padres e hijos, alumnoseducadores y padres-profesorado. El modelo de intervención seguido se puede considerar ecléctico: enfoque instructivo y social, informando, trabajando en grupo, analizando casuísticas, exponiendo experiencias, buscando respuestas y proponiendo alternativas a los temas tratados. Los contenidos se encuadran dentro de tres bloques: higiene mental y desarrollo de la personalidad, educación para la salud y aspectos educativos y escolares. El grado de satisfacción tanto a padres como a profesores -desprendido de la evaluación de la experiencia- ha llevado a la continuación de la misma tratando de incentivar uno de los aspectos que tanto se reclama en los contextos educativos para conseguir una enseñanza de calidad: la colaboración y participación de las familias en el seno de la vida escolar.
\end{abstract}

Palabras clave: Colaboración escuela-familia, escuela de padres.

\begin{abstract}
It was intended to promote the communication and dialogue with the present experience in a permanent and comprehensive way between the teachers and families in our educational enviroment. The
\end{abstract}

* Licendiado en Ciencias de la Educación y Diplomado en Profesorado de E.G.B. En la actualidad trabaja como orientador escolar. 
establisment of the projet was coordinated by the school pedagogical specialist relying on the support and colaboration of the school management and also of a group of tenchers and families who worked on this projet.

A period reflexion was created to study daily conflicts in order to offer solutions, alternatives, norms for action and to achieve a better understanding betiveen parents and childreu, students and educators, parents and teachers. The patterns for intervention followed was ecletic: social and instructive focus with a lot of information and group work analysing individual cases, sharing experiences, looking for auswers and proposing alternative solutions to the subjet dealt with. The contents can be placed into three categories: mental hygiene and personal developmant, headth education and school issues. The lord of satisfaction for both the parents and the teachers -according to the control of the experiencehas brought about the continuation of this experience trying to encourage oncof the desired goals so demanded ley the educational projects to achieve a teaching of quality: the family cooperation and participation in the school life.

Key words: School-families cooperation, parents school.

\section{Descripción del proyecto}

Las funciones educativas de la familia y de la escuela se complementan y es necesario que se coordinen para integrar los variados influjos educativos que el ambiente social de la zona puede ir introduciendo.

La comunicación continuada y comprensiva entre padres y educadores es fundamental en todo el proceso educativo. Los padres tienen el derecho de conocer la marcha del proceso en relación con sus hijos y los educadores deben tener presente esta relación en todo momento. Es imprescindible que ambas parte (familia y escuela) funcionen de una forma coordinada, aunando objetivos y multiplicando esfuerzos.

El contacto de los padres con la escuela no acaba, ni mucho menos en la elección de la misma -independientemente de que haya sido esta elección obligatoria o voluntaria-. Tampoco debe basarse en continuas y reiteradas visitas al profesor. Centro y padres deben facilitar el que se produzcan espacios de reflexión conjunta sobre problemáticas de hijos y alumnos, y como un deseo mutuo de acercamiento y escucha, que sirva para generar cambios y prever futuras desadaptaciones.

En el preámbulo de la Ley Orgánica de Ordenación General del Sistema Educativo se afirma:

«Ninguna reforma consistente, tanto más si se trata de la educativa, puede arraigar sin la activa participación social.

Particularmente relevante para la consecución de sus objetivos es la participación de los distintos sectores de la comunidad educativa, singularmente de los padres, profesores y alumnos. Esta participación, consagrada y regulada en nuestro ordenamiento jurídico, se verá fomentada en el marco de esta reforma y se recogerá en los distintos tramos y niveles del sistema educativo ...».

De un modo muy resumido diremos que la colaboración entre padres y educadores es indispensable por las siguientes razones:

- Porque la educación se da antes que nada, en el seno familiar y los padres no pueden estar ajenos al objetivo común que les une con la escuela: la educación de sus hijos. 
- Porque los hechos que se desarrollan en la escuela son objetos de comunicación para la sociedad y las familias directamente implicadas.

- Porque la educación no es un hecho aislado sino dentro de un sentido de responsabilidad compartida en cooperación y por medio de una activa participación conjunta en los procesos educativos.

- Porque siente y percibe favorablemente la participación de los padres en la escuela, aportándoles seguridad, incentivos y modelos para la propia socialización.

- Porque la participación de los padres en el centro transforma tanto la experiencia familiar como la escolar, reuniendo en un esfuerzo conjunto la formación del niño desde un punto de vista íntegro, consolidando diferentes formas de comunicación.

Nuestro centro está situado en una barriada del extrarradio de la ciudad cuyos alumnos proceden de un ambiente sociocultural bajo o muy bajo. En general, la actitud de los padres es un tanto pasiva, sobre todo ante la preocupación que manifiestan por las cuestiones escolares y la participación y colaboración con el centro escasea, acudiendo esporádicamente cuando se les cita y, a veces, tampoco acuden, o bien cuando deciden voluntariamente acudir para hablar con los tutores de sus hijos o el orientador del centro. Sólo se consigue un porcentaje más elevado de asistencia cuando se organizan asambleas informativas al inicio de cada curso o bien al final de cada uno de los trimestres, coincidiendo con la entrega de los resultados de cada una de las evaluaciones - citados previamente-. En este sentido, queda más que justificada la implantación de esta experiencia que tan necesaria la entendemos para el desarrollo curricular y la educación de nuestros escolares.

La iniciativa surge del Departamento de Orientación y un colectivo de profesores del propio centro, respaldada y apoyada por la Dirección del mismo, siendo el departamento anteriormente citado y más concretamente el Orientador quien se encargue de coordinar y dinamizará todo el proceso: diseño, desarrollo y valoración del proyecto.

\section{Grado de consecución de los objetivos}

En cuanto a los propósitos o metas más globales que pretendíamos, la principal era acercar y atraer a las familias a la escuela de un modo más permanente y continuo; y que no tuvieran la oportunidad exclusivamente cuando se les citaba de una manera puntual coincidiendo con las sesiones de evaluación o situaciones esporádicas en las que se reclamaba su presencia en el centro con el fin de buscar alternativas o respuestas conjuntamente.

Por otro lado, se pretendía que toda esta labor desempeñada por el grupo de profesores y familias colaboradoras en el desarrollo del plan tuviera, de alguna manera, y en la medida de lo posible, cierta repercusión en apoyar la labor educativa conjuntamente tanto por parte del profesorado dentro de las aulas como dentro del propio seno familiar.

De manera más concreta y específica podemos afirmar que los logros a corto plazo alcanzados se pueden resumir en los términos siguientes:

- Se ha fomentado el diálogo en general y se ha disfrutado de una nueva experiencia desconocida hasta entonces en nuestro contexto educativo. 
- Se ha logrado una información y formación específica en el tratamiento de cada uno de los bloques temáticos desarrollados para el desempeño del papel de los padres como propios educadores de sus hijos/as.

- La experiencia ha permitido en mucha medida aprender a trabajar en grupo y a participar individual y colectivamente dentro de un orden establecido.

- No queda al margen el ofrecimiento de modelos alternativos de actuación de las familias para el trato y la educación de sus hijos/as de un modo real, útil y funcional.

- Se han creado actitudes positivas y tolerantes a lo largo del desarrollo de los distintos planteamientos puestos en práctica.

- Se han analizado problemas y se han compartido experiencias mutuamente.

- Todo ello ha sido posible, además gracias a la potenciación y coordinación para que el funcionamiento de la Escuela de Padres fuera una realidad.

Esperemos que a medio plazo la experiencia haya servido para crear un espacio de reflexión sobre los conflictos cotidianos surgidos dentro de las familias y que tienen su repercusión y continuidad en la labor educativa dentro de nuestro centro educativo.

Deseamos así mismo, que el entendimiento ente familias y profesorado, padres e hijos y profesores-alumnos sea cada vez más fluido y real.

A largo plazo se pretende generar cambios y prever futuras desadaptaciones en aquellos casos en que pudieran producirse.

\section{Contenidos desarrollados}

El desarrollo de los contenidos ha seguido el curso y temporalización prevista en el proyecto inicial, si bien han sufrido una cierta modificación (supresión de algún tema e incorporación de otros). La tabla 1 refleja los bloques de contenido y temas desarrollados:

\section{Descripción de los materiales elaborados}

Los materiales manejados en cada una de las sesiones hacían referencia a los siguientes parámetros:

A) Material básico diseñado por cada uno de los ponentes para el desarrollo del plan específico del tema (transparencias, guiones, actividades, casuísticas para analizar, etc.).

B) Material complementario para el desarrollo íntegro de cada tema comprado o aportado por el Departamento de Orientación o por los propios profesores ponentes (vídeos, bibliografía, material farmacéutico, etc.).

C) Material propiamente elaborado por el profesorado para las familias, ofreciendo pautas y modelos alternativos de actuación para la educación de sus hijos/as como padres (folletos, viñetas, dípticos, trípticos, etc.).

D) Material complementario para las familias que sirviera de anexo informativo y de un modo más exhaustivo y detallado sobre la temática desarrollada. En este sentido han 


\section{TABLA 1.}

\begin{tabular}{|l|l|}
\hline \multicolumn{2}{|c|}{ 1. Higiene mental y desarrollo personal } \\
\hline 1.1. & La adolescencia. \\
\hline 1.2. & Aspectos psicológicos. \\
\hline 1.3. & Implicaciones sociales. \\
\hline 1.4. & La autoestima. \\
\hline \multicolumn{2}{|c|}{ 2. Educación para la salud } \\
\hline 2.1. & Higiene bucodental: una apuesta por la calidad de vida. \\
\hline 2.2. & Alimentación y nutrición: la dieta en edad escolar. \\
\hline 2.3. & Introducción al mundo de la drogadicción: alcohol y tabaco. \\
\hline 2.4. & Anorexia y bulimia. \\
\hline \multicolumn{2}{|c|}{ 3. Aspectos educativos y escolares } \\
\hline 3.1. & Actitud de la familia ante el estudio de sus hijos/as. \\
\hline 3.2. & Actitud de la familia ante el fracaso escolar. \\
\hline 3.3. & El uso y abuso de la tv. \\
\hline 3.4. & La lectura como base del aprendizaje. \\
\hline 3.5. & Las lecturas de tus hijos/as. \\
\hline 3.6. & Los valores, normas y actitudes también son tarea de la familia. \\
\hline 3.7. & La familia como modelo de comportamiento: la educación no sexista. \\
\hline 3.8. & El diálogo y la comunicación como base de las relaciones humanas y familiares. \\
\hline
\end{tabular}

colaborado organismos como El Comisionado para la Droga, El Ayuntamiento, Asuntos Sociales, etc.

Estos dos últimos bloques se anexan en un dossier para entrega del Centro de Profesores como elaboración propia y complementaria destinada a las familias, cuyo título diseñado para la ocasión ha sido: Información y Asesoramiento familiar: pautas orientativas y modelos alternativos de actuación.

\section{Análisis de la dinámica de trabajo del grupo}

En este sentido la dinámica del grupo ha trabajado en dos vertientes diferenciadas:

- Por un lado existían sesiones de coordinación general por parte del Jefe del Departamento de Orientación en las que se aportaban y trataban, entre otras, temas como:

- Puesta en marcha de la Escuela de Padres.

- Proceso de formación. 
- Fases:

* Sensibilización por parte del profesorado (existía desde hacía ya tiempo).

* Concienciación y sensibilización por parte de los padres.

* Composición del grupo por parte del profesorado: once en total pertenecientes a los departamentos:

- Orientación: 2 profesores (desarrollo de 5 temas).

- Lengua y Literatura: 3 profesores

(desarrollo de tres temas).

- Matemáticas: 1 profesora (desarrollo de un tema).

- Ciencias Naturales: 3 profesores

(desarrollo de dos temas).

- Ciencias Sociales: 1 profesor (desarrollo de un tema).

- Religión: 1 profesor (desarrollo de un tema).

- Educación Física: 1 profesora (desarrollo de un tema).

* Composición del grupo de familias destinatarias:

En un principio el planteamiento iba dirigido a familias cuyos hijos/as estaban escolarizados en el Primer Ciclo de la ESO pero posteriormente se extendió también a familias cuyos hijos/as estaban matriculados en el Segundo Ciclo de ESO y familias cuyos hijos/as estaban escolarizados en el nivel de Primaria.

Para su composición se utilizaron asambleas informativas, reuniones por niveles educativos, envío de circulares y comunicados.

- Delimitación de los temas.

- Elaboración del Proyecto.

- Implementación.

- Valoración y evaluación.

- Guiones de trabajo.

- Material y bibliografía.

- Marcha de las sesiones.

- Grado de asistencia de las familias.

- Temas que iban surgiendo paralelamente y en función de las sesiones de coordinación por parte del Departamento de Orientación.

En la delimitación y selección de los temas se tuvo en cuenta los siguientes factores y variables:

- Detección de necesidades por parte de las familias.

- Motivaciones e intereses propios y generalizados por quienes se comprometieron a asistir.

- La edad del alumnado de nuestro centro educativo en función de la selección y priorización de los distintos bloques de contenido. 
- Por parte del profesorado, que tuvo en cuenta lo anterior, era necesario la intervención directa con las familias para el desarrollo de las temáticas puestas en práctica, así como habilidades cognitivas en las mismas y cierta formación al respecto.

- Por otro lado, las distintas sesiones de intervención se venían desarrollando ofreciendo a través de la técnicas y estrategias:

- Información y asesoramiento.

- Utilización de debates dirigidos.

- Análisis y comentario de casos.

- Intercambio de experiencias.

- Trabajo en grupo.

- Establecimientos de pautas orientativas y ofrecimiento de modelos alternativos de actuación familiar de un modo real, útil y funcional.

- Otro tipos de estrategias y técnicas utilizadas en cada sesión.

\section{Grado de implicación de los componentes del grupo}

El grado de implicación del grupo de profesores, desde un primer momento ha sido total y pleno. El hecho de que se formara un grupo de carácter voluntario con iniciativas propias de actuación e intervención ha hecho posible el pleno desarrollo de nuestro plan de trabajo.

La concienciación y sensibilización por el tema desde hacía tiempo también han hecho posible un buen efecto y viabilidad de las propuestas diseñadas.

En cuanto a las familias, hay que decir que la implicación en cada una de las sesiones ha sido bastante satisfactoria, si bien a nivel global podemos decir que el grado de asistencia fue más elevado en las primeras sesiones desarrolladas.

Dentro de este apartado se comentó más detenidamente algunas propuestas para potenciar la asistencia de mayor números de familias a este tipo de actuaciones tan importantes y necesarias.

\section{Otros factores que han hecho posible el proyecto}

\section{Espacios y tiempos}

Se habilitaron dos aulas en el IES TORREBLANCA de Sevilla. Una para el servicio de guardería y otra para el desarrollo de los distintos temas con las familias.

El horario fue de 18 a 20 horas, sobrepasando algunas veces este espacio temporal.

Las sesiones se impartían los martes. El comienzo fue el 10 de Noviembre y el final el 13 de Abril. Se desarrollaron un total de 15 sesiones. 


\section{El servicio de guardería}

Para las familias que tenían necesidad de asistir a la Escuela de Padres y, al mismo tiempo no disponían de medios para dejar a sus hijos menores con alguien que cuidara de ellos, se habilitó un servicio de guardería en el propio centro que pudiera desempeñar la labor de cuidado de estos niños.Este servicio fue organizado por el profesorado y en donde prestaban ayuda y colaboración voluntaria el alumnado de $4^{\circ}$ de ESO y Bachillerato de un modo rotativo y siempre por parejas.

Al respecto se trató además que durante ese tiempo de permanencia en el centro, los niños menores tuvieran la oportunidad de aprovechar el tiempo de una manera más lúdica y divertida, pintando, escribiendo, dibujando o simplemente jugando. Para ello se compraron materiales como bolígrafos, lapiceros de colores, papel continuo, ceras, rotuladores y otro material complementario en función de las actividades a realizar.

\section{Registro y grado de asistencia}

En cada una de las sesiones se registraba la asistencia marcando una cruz en la casilla donde aparecía el nombre y los apellidos del alumno cuyos padres asistían. Las listas se confeccionaron desde un principio cuando se procedió a realizar un sondeo sobre las personas interesadas en asistir a la Escuela de Padres. A esas listas se fueron añadiendo nuevas familias que, en un principio no empezaron a asistir.

Durante las primeras sesiones las expectativas se habían hecho realidad, la asistencia estuvo asegurada sobre una treintena de familias, número estimado desde un principio del funcionamiento y organización. En las últimas sesiones bajó el número de asistentes, debido principalmente a que el desarrollo de las sesiones no era lo suficientemente continuo - una sesión por semana- y a cierta interrupción que sufrió el calendario escolar por coincidir con fechas señaladas como Semana Santa o feria.

Sobre las causas de este absentismo no se ha profundizado en exceso pero entre los factores que se aluden están no tanto la desmotivación como la priorización de otras tareas interesantes también para el hogar; para algunas personas coincidía el espacio temporal todavía con la jornada laboral o bien con el término de la misma y venían muy cansados; la semana que no había parece como si se perdiera el hilo conductor.

En las primeras sesiones se dejaron ver parejas completas correspondientes a algunas familias (ambos cónyuges). Pronto empiezan a desaparecer los varones, por lo que la asistencia en la gran mayoría de los casos estaba constituida por madres, lo que nos hace pensar todavía que la educación, en el mejor de los casos, al menos en nuestro contexto educativo, sigue estando relegada a las madres; y si éstas no tienen tiempo de frecuentar este tipo de actividades difícilmente va a ser suplida su asistencia por el otro cónyuge.

Desde un principio, y así se contemplaba en nuestro proyecto, difícilmente se podrán prever conductas desadaptativas o producir cambios si los dos cónyuges no van en la misma dirección de cara a la educación de sus hijos. Son actividades, tareas y compromisos que tienen que asumir con verdadera responsabilidad los padres, porque son los dos quienes deben educarlos. 


\section{Modalidad de las sesiones}

Se optó por el acotamiento de los temas, dándoles principio y fin en cada una de las sesiones, exceptuando el tema de las actitudes, valores y normas que continuó durante dos sesiones. El motivo venía justificado por el compromiso de intervenir desarrollando todos los temas que se habían previsto.

\section{Registro idiomático empleado}

Nos situamos en un contexto sociocultural y económico muy bajo, bajo o medio bajo por lo que el profesorado tenía hábito en esta forma de proceder tanto con las familias como con el alumnado.

Por ello, desde un principio esta preocupación estuvo presente y se convirtió en una condición sine qua nom para poder implementar los temas. Se estuvo observando en todo momento que los mensajes, las informaciones y demás actividades desarrolladas estaban siendo asimiladas de un modo permanente y continuo. Prueba de ello es el grado alto de participación por parte de las personas asistentes.

\section{Evaluación de la escuela de padres}

En este apartado vamos a distinguir tres apartados diferenciados, a saber:

- Criterios generales y particulares de evaluación y valoración.

- Las técnicas e instrumentos de recogida de datos generales del planteamiento y en cada una de las sesiones de intervención con las familias.

- Elaboración de conclusiones.

\section{A. Criterios generales de valoración}

A.1. Interés de los asistentes.

A.2. Grado de motivación y participación.

A.3. Grado de asistencia.

A.4. Efecto/viabilidad en el trato con los hijos/as.

A.5. Grado de funcionalidad y utilidad de los temas tratados.

A.6. Novedades que aporta cada tema.

A.7. Claridad en el planteamiento.

A.8. Respuestas o modelos alternativos que ayudan en la práctica diaria en el trato con los hijos/as.

A.9. Valoración recursos empleados.

A.10. Dinámicas trabajadas.

A.11. Otros. 


\section{B. Técnicas e instrumentos de recogida de datos}

B.1. Charlas y coloquios.

B.2. Asambleas y reuniones por niveles educativos.

B.3. Circulares, comunicados, ...

B.4. Debates.

B.5. Entrevistas individuales.

B.6. Cuestionarios ad hoc para profesorado y familias.

B.7. Informes.

B.8. Escalas de registro asistencial.

B.9. Análisis de casuísticas.

B.10. Memoria.

B.11. Otros.

\section{Conclusiones extraídas por el profesorado}

- Se califica la experiencia como muy gratificante para todos los/as profesores/as que han intervenido con las familias.

- La respuesta, en general, dada por las familias se puede considerar buena y muy satisfactoria, en cuanto al grado de colaboración y participación de las personas asistentes a las distintas sesiones.

- Sobre la asistencia hay que confirmar que en la mayoría de las sesiones ha sobrepasado más de la mitad de familias de las que se esperaban. Sigue siendo éste, un factor importante a potenciar en futuras experiencias. El grado de asistencia disminuye, como se ha señalado anteriormente, en las últimas sesiones de intervención.

- Se puede decir que el contenido, las informaciones o el material entregado a las familias ha conectado altamente con sus necesidades, intereses y expectativas.

- El grado de satisfacción percibido ha sido alto o muy alto.

- Todo el profesorado está dispuesto a repetir la experiencia, introduciendo cambios o modificaciones pertinentes, susceptibles de madurar y concretar, entre las que se encuentran:

- Posibilidad de intervención de dos horas semanales con una duración de una hora aproximadamente.

- Profundizar y dar un tratamiento más exhaustivo a ciertas temáticas.

- Posibilidad de asistencia conjunta a algunas sesiones de hijos/as y familias.

- Introducir los temas que las personas asistentes vean conveniente y necesario. 


\section{Conclusiones extraídas por las familias}

- Todos los temas han estado conectados en mayor o menor medida con sus intereses, motivaciones y necesidades.

- Es cierto que ha habido personas que hubieran deseado el desarrollo de temas no tratados como las relaciones de pareja, la educación sexual o la orientación vocacional y profesional de sus hijos: salidas formativas y académicas.

- El desarrollo, en general, del planteamiento ha supuesto una cierta incidencia en la mejora de las relaciones con los hijos/as dentro de la familia.

- Se afirma la claridad con que han sido tratados los temas, procurando hacerlo con un lenguaje sencillo y directo, fácil de asimilar.

- Los materiales empleados, así como los recursos y medios utilizados han parecido muy interesantes y adecuados a los temas tratados (transparencias, casos, folletos, dípticos, vídeos, etc.).

- El grado de participación se puede considerar alto.

- Se considera que el grado de aplicabilidad, utilidad y funcionalidad de lo aprendido es alto en la gran mayoría de las ocasiones.

- Sobre el grado de asistencia las familias sugieren la creación de mecanismos -al igual que el profesorado- para potenciar su presencia y optimizar, en cierto sentido los recursos humanos:

- Lanzar circulares previas a cada tema.

- Servirse de las mismas familias asistentes para difundir la información.

- Anunciar publicitariamente en la emisora local de TV. o radio.

- Elaboración de carteles, pegatinas, ping, o similares, como estímulos más fuertes para atraer al personal.

- Contar también con la colaboración del propio alumnado.

- Se puede dar una valoración global altamente positiva, hasta el punto de que este tipo de experiencias tenían que haberse dado mucho antes y debieran estar generalizadas en todos los centros educativos.

\section{Bibliografía}

Bellido, C. (1983): Problemática en la comunicación padres-hijos adolescentes. Narcea. Madrid.

Bertranquer, M. (1981): Escuela de padres. Herder. Barcelona.

Caparrós, M. (1977): Crisis de la familia. Fundamentos. Madrid.

De Vega y Relea (1975): La familia y la educación: orientaciones pedagógicas para los padres en la formación de los hijos. Escuela Española. Madrid.

Debesse, M. (1977): La adolescencia. Oikos-Tau. Barcelona.

Díez, J. J. (1983): Familia-escuela: una relación vital. Narcea. Madrid.

Fabré, N. (1972): La educación familiar y sus problemas. Marova. Madrid. 
Ibáñez López, P. y Sanjuan, A. M. (1983): La droga. Razones de su consumo para la juventud. Mezquita. Madrid.

Isaacs, S. (1974): Conflictos entre padres e hijos. Psique. Barcelona.

Isambert, A. (1980): La educación de los padres. Herder. Barcelona.

Jackson, B. (1981): Cada niño una excepción. Morata. Madrid.

Jersild, A. T. (1972): Psicología de la adolescencia. Aguilar. Madrid.

Lacan, J. ((1970): La familia. Fundamentos. Madrid.

Lafay, J. B. (1983): Cómo hacer felices a los niños. Narcea. Madrid.

Laing, R. D. (1979): Conversaciones con mis hijos. Crítica. Barcelona.

López Nomdeu, C. (1981): La alimentación: base de la salud. Narcea. Madrid.

Lynch, J. y Pimlott, J. (1979): Padres y profesores. Anaya. Madrid.

Martínez, B. (1981): La familia ante el fracaso escolar. Narcea. Madrid.

Miraldelll, E. (1977): ¿Sabemos ser padres? Magisterio Español. Madrid.

Moratinos, J. F. (1985): La Escuela de Padres. Narcea. Madrid.

Movilla, S. (1982): Los jóvenes se drogan... ¿Qué hacer? Narcea Madrid.

Navarro, M. (1982): Análisis sociológico de la familia española. Ministerio de Cultura. Madrid.

Norte, M. (1982): Sólo educa el que ama. Narcea. Madrid.

Olive y Cooper: La educación y las actitudes de los padres. Paidós. Buenos Aires.

Pallarés, M. (1982): Técnicas de grupo para educadores. Publicaciones ICCE.

Peine, H. y Howarth, R. (1985): Padres e hijos: problemas cotidianos de conducta. Pablo del Río. Madrid.

Percival, S. y otros (1974): Las relaciones familiares. Paidós Buenos Aires.

Piaget, J. y cols (1980): El desarrollo del adolescente. Horme. Buenos Aires.

Piedrola (1978): Medicina preventiva y social. Amaro. Madrid.

Pumarola Busquets, A. y cols. (1983): Medicina preventiva y social. Higiene y sanidad ambiental. Madrid.

Ridao, I. (1984): Tesis doctoral (inédita): Influencia de la familia en el Rendimiento Escolar. Universidad de Sevilla.

Riesgo, L. y Pablo, C. (1982): Los puntos claves de toda educación. Narcea. Madrid.

Ríos González, J. A. (1972): Familia y centro educativo. Paraninfo. Madrid.

Ríos González, J. A. (1978): Fracaso escolar y vida familiar.

Marciega. Madrid.

Ríos González, J. A. (1980): El padre en la dinámica personal del hijo. Científico-Médica. Barcelona.

Ríos González, J. A. (1983): Crisis familiares: causas y repercusiones. Narcea. Madrid.

Rocheblave-Spenle, A. M. (1980): El adolescente y su Mundo.

Herder. Barcelona.

Rof Carvallo, J. (1980): La familia: diálogo recuperable. Karpos. Madrid.

Scekel (1973): La educación de los padres. Paidós. Buenos Aires.

Schofied, M. (1976): El comportamiento sexual de los jóvenes. Fontanella. Barcelona.

Soifer, R. (1979). ¿Para qué la familia? Kapelusz. Buenos Aires.

Soto, F. (1982): Actitudes familiares: cuándo son problema y cómo cambiarlas. Narcea. Madrid. 
Stierlin, H. y otros (1981): Terapia de familia. Gedisa. Barcelona.

UNESCO (1971): Educación para la salud, el sexo y la familia. Paidós. Buenos Aires.

Urdiales, C. (1981): ¿Qué transmitir hoy a nuestros hijos? Narcea. Madrid.

Varios (1980): Relación familiar padres e hijos. Karpos. Madrid.

Vivanco, F., Palacios, J. M., García Almansa, A. (1976): Alimentación y nutrición. Dirección General de Sanidad. Programa EDALNU. Madrid.

Waklroos, S. (1978): La comunicación en la familia. Diana. México.

Weil (1974): Relaciones humanas entre los niños, los padres y sus maestros. Kapelusz. Buenos Aires. Zulliger, H. (1977): Niños dificiles. Morata. Madrid.

Fecha de recepción: 14-II-01

Fecha de revisión: 10-IV-01

Fecha de aceptación: 16-X-01 